\title{
Psychological factors affecting the association between a lifestyle behavior intervention and depressive symptoms in low-income overweight and obese mothers with young children: a secondary data analysis
}

Lorraine B. Robbins ( $\sim$ robbin76@msu.edu )

Michigan State University College of Nursing https://orcid.org/0000-0003-2914-3630

Mei-Wei Chang

Ohio State University

Jiying Ling

Michigan State University

Roger Brown

University of Wisconsin Madison

Research article

Keywords: Depression, Motivation, Emotions, Female, Women, Obesity

Posted Date: July 27th, 2020

DOI: https://doi.org/10.21203/rs.3.rs-44249/v1

License: (9) This work is licensed under a Creative Commons Attribution 4.0 International License.

Read Full License 


\section{Abstract}

Background Psychological factors mediating the effect of lifestyle behavior interventions on mothers' depressive symptoms are unclear. Using data from a community-based lifestyle behavior intervention, this secondary analysis examined whether autonomous motivation, coping self-efficacy, and emotional coping mediated the association between the intervention and depressive symptoms in low-income overweight and obese mothers with young children.

Methods This 16-week intervention had 2 components: Mothers watched 10 video lessons in Digital Video Disc (DVD) format at home and joined 10 peer support group teleconferences (every week in weeks 1-4; then every other week in weeks 5-16). All participants completed reliable and valid measures of autonomous motivation, coping self-efficacy, emotional coping and depressive symptoms via phone at baseline and immediately after the intervention. To assess effect size, proportion of maximum possible (POMP) scores were calculated. To test mediation effects, composite indicator structural equation modeling was performed.

Results The intervention significantly alleviated depressive symptoms ( $p=0.015, \mathrm{POMP}=-4.31 \%)$. While the intervention had no influence on autonomous motivation, it significantly increased coping selfefficacy $(p<0.001, \mathrm{POMP}=7.4 \%)$ and emotional coping $(p=0.003$, POMP $=3.81 \%)$. Coping self-efficacy significantly and negatively mediated the association between the intervention and depressive symptoms $(p=0.002, \mathrm{POMP}=-2.53 \%)$. However, autonomous motivation and emotional coping were not significant mediators.

Conclusions To help this target population alleviate depressive symptoms, clinicians may consider teaching these women practical strategies that can be applied to daily life to boost coping self-efficacy.

\section{Background}

Maternal depression is a major global public health problem [1], especially for low-income mothers of young children [2-5] with as high as $50 \%$ suffering from this condition [6]. Maternal depression, which is characterized by feelings of helplessness, isolation, guilt, and hopelessness [7], is a serious morbidity for both mother and child and strong predictor of mothers' suicide [1]. Adverse effects on child well-being [8] include behavioral problems, delayed emotional development [2], increased stress, weakened immune system, and impaired maternal and child relational behaviors [9]. To prevent this negative trajectory of child outcomes, helping mothers with young children to prevent or reduce depressive symptoms is critically important [10].

Sadly, a higher percentage of low-income mothers with major depression who have young children receive no treatment for the condition, as compared to a similar group of higher-income mothers (37.3\% versus $25.3 \%$, respectively) [11]. Also, low-income mothers who are overweight or obese are more likely to report depression than those with a healthy weight and higher income [2-5]. Moreover, many low-income mothers without depression in the early postpartum period can become depressed a few years later [12], 
and depression among overweight or obese mothers can continue across several years after giving birth [13-15]. Yet, interventions to alleviate depressive symptoms have been limited to the perinatal period [15] and have not included low-income overweight and obese mothers with young children, who are a vital priority for intervention.

Fortunately, maternal depression is preventable via healthy lifestyle behavior interventions [16]. In a systematic review and meta-analysis of five randomized controlled trials (RCTs) investigating healthy lifestyle behavior interventions promoting physical activity (PA) and/or nutrition in overweight or obese women of reproductive age, van Dammen and colleagues [16] found that the interventions reduced depressive symptoms. Unfortunately, only two of the five interventions were theory-based, and no analysis was conducted in any of the studies to identify whether any theoretical variables mediated the effect of the intervention on depressive symptoms.

Three theoretical variables, including autonomous motivation, coping self-efficacy, and emotional coping, have been linked to mental health outcomes. Autonomous motivation, which is defined as an inner drive to engage in a behavior because of personal value, interest, or choice, is a key concept of the SelfDetermination Theory [17]. Increased autonomous motivation for participating in a PA intervention has been associated with fewer depressive symptoms in middle-aged overweight and obese women [18]. Selfefficacy and emotional coping are key concepts of the Social Cognitive Theory [19]. Self-efficacy in general refers to the confidence that individuals have in their abilities to successfully perform various functions [19]. An inverse association between generalized self-efficacy and depressive symptoms has been demonstrated in depressed low-income mothers of all body sizes with children aged 6 weeks to 36 months [20]. Coping self-efficacy, defined as individuals' confidence in their ability to cope with stressors, threats, and challenges [21], has been found to have a strong negative association with depression in female college students [22]. Emotional coping, which refers to strategies used to deal with negative feelings, has been negatively related to depression in caregivers of children with cerebral palsy [23]. Although evidence has shown associations between depressive symptoms and autonomous motivation, coping self-efficacy, and emotional coping, whether these concepts serve as mediators in lifestyle behavior interventions to alleviate depressive symptoms remains unknown.

Identifying the theoretically informed mediators that underly the alleviation in depressive symptoms from lifestyle behavior interventions (process of change or mediation effect) in varied populations is important so that relevant psychological factors can be efficiently targeted to promote intervention success.

Findings from a recent RCT testing a 16-week lifestyle behavior intervention in low-income overweight and obese mothers with young children has shown an intervention effect on coping self-efficacy $(p \leq$ 0.01, effect size [Cohen's $d$ ] $=0.53,95 \% \mathrm{Cl}: 0.31-0.76)$, emotional coping ( $p \leq 0.01, d=0.38,95 \% \mathrm{Cl}:-0.49$, -0.04 ), and depressive symptoms ( $p<0.05, d=-0.27,95 \% \mathrm{Cl}:-0.48,-0.04)$ immediately after the intervention [24]. However, the intervention effect on autonomous motivation and potential theoretical mediators was not examined. To address this gap and extend beyond the finding, this secondary analysis using data from the trial investigated whether autonomous motivation, coping self-efficacy, and emotional coping mediated the association between the intervention and depressive symptoms in low- 
income overweight and obese women with young children. It is hypothesized that the intervention would increase autonomous motivation, coping self-efficacy, and emotional coping; and then translate into an alleviation of depressive symptoms.

\section{Methods}

\section{Participants and setting}

The study procedure for the RCT testing the 16-week lifestyle behavior intervention was approved by the Michigan State University and Michigan Department of Community Health Institutional Review Boards. Women were recruited from The Special Supplemental Nutrition Program for Women, Infants, and Children (WIC) [25] in Michigan while waiting for their appointments. WIC is one of the largest federally funded nutrition programs in the U.S. and serves low-income women who are pregnant, postpartum and breastfeeding; and children from $0-5$ years of age. WIC serves individuals with annual household income at or below $185 \%$ of the federally poverty line [25].

From 2012-2015, trained peer recruiters invited women to be screened and then measured each potential participant's height and weight to compute body mass index (BMI). To be eligible to participate, women had to have a BMI of 25.0-39.9 kg/m², be non-Hispanic Black or White, be between 18-39 years old, and have a biological child between 6 weeks and 4.5 years old enrolled in WIC. All women meeting eligibility criteria provided written consent prior to participation in the study [26-27]. A detailed CONSORT participant flow diagram has been published previously [27].

\section{Intervention}

Details regarding the intervention lessons have been published elsewhere [26]. Briefly, the intervention was 16 weeks in duration and covered topics of stress management (four weeks), healthy eating (five weeks), and physical activity (one week). During the 16-week intervention, intervention participants joined 10 peer support group teleconferences (30-minute/session every week in Weeks 1-4 and then every other week in Weeks 5-16) directed toward enhancing autonomous motivation. WIC dieticians and peer educators, who were trained in motivational interviewing, led the group discussion. Motivational interviewing is a method of communication directed toward eliciting an individual's motivation, especially autonomous motivation [28], for making positive behavioral changes [29]. Intervention participants also watched 10 video lessons in Digital Video Disc (DVD) format at home (20 minutes/lesson every week in Weeks 1-4 followed by every other week in Weeks 5-16). The videos applied concepts of coping selfefficacy, emotional coping, and observational learning processes by showing role models of four peers of the study population. The use of peers enabled participants to relate to role models and observe their positive lifestyle behaviors [19]. The peers demonstrated a variety of practical skills to help viewers choose what they could do to develop feelings of competence and identify their strengths to build coping self-efficacy for positive behavior change. They also showed effective ways to respond to negative emotions to improve emotional coping. 


\section{Measures}

Data were obtained from 338 women who completed phone interviews designed to assess autonomous motivation, coping self-efficacy, emotional coping, and depressive symptoms at baseline (Time [T]1) and immediately after the 16-week intervention (Time [T] 2). Trained interviewers, who were blinded to group allocation in the RCT, conducted the phone interviews and simultaneously entered data into a data collection system created for the trial.

Demographics. Using a pencil-and-paper survey, women provided demographic data regarding their race, education, employment and smoking status at the screening. Age and postpartum period were calculated based on each woman's and her youngest child's date of birth, respectively.

Autonomous motivation. The validated Treatment Self-Regulation Questionnaire (six items; Cronbach's alpha coefficient $[\mathrm{a}]=0.92$ ) was used to assess autonomous motivation [30]. Women were asked about what motivated them to regularly engage in positive behaviors. For example, one reason was, "because I want to take responsibility for my own health." Response choices ranged from 1 (not at all true) to 7 (very true). A higher mean score for the six items indicates higher autonomous motivation.

Coping self-efficacy. A 10-item survey with established construct validity and good reliability (Cronbach's $a=0.92$ ) was used to measure coping self-efficacy. Women were asked about their confidence in or belief about their ability to successfully engage in positive behavior or manage obstacles or challenges. A survey item example was "You can relax, even when you have too much to do." Response choices ranged from $1=$ not at all confident to $4=$ very confident. A higher mean score for the 10 items indicates higher coping self-efficacy [24].

Emotional coping. A validated 5 -item survey (Cronbach's $a=0.91$ ) was used to measure emotional coping or the frequency that appropriate strategies are used to manage emotions or stress. One example of a survey item was "How often do you deal with or prevent stress by talking to family members?" Response choices ranged from $1=$ rarely or never to $4=$ usually or always. A higher mean score for the five items indicates better emotional coping [31].

Depressive symptoms. The 20-item Center for Epidemiologic Studies Depression Scale (CES-D) with concurrent validity and reliability (Cronbach's $a=0.85-0.90$ ) [32] was used to measure depressive symptoms. Response choices ranging from 0 (rarely or none of the time) to 3 (most or all of the time) were summed. A higher score indicates more depressive symptoms [32].

\section{Statistical analysis}

Mplus (version 8) was used to conduct all statistical analyses. Intervention was the exogenous variable (independent variable or predictor). The endogenous variable (outcome or dependent variable) was depressive symptoms. Mediators included autonomous motivation, coping self-efficacy, and emotional coping. Covariate was postpartum period, which has been associated with depressive symptoms [33]. The Composite Indicator Structural Equation (CISE) modeling using maximum likelihood estimation [34] 
was performed to examine mediation effects while adjusting for baseline measurements and postpartum period. As shown in Fig. 1, the mediation model tested a system with four equations simultaneously: one endogenous variable (depressive symptoms) and three mediators (autonomous motivation, coping selfefficacy, and emotional coping).

CISE modeling is an errors-in-variables regression approach that models measurement errors in both the exogenous and endogenous variables [35]. CISE modeling creates latent variables by combining items of each separate measurement domain into a single indicator [34]. To control for measurement errors in a CISE model, the error variance of the indicator was fixed at $(1-a)^{\star} \sigma^{2}$, where a was Cronbach's alpha, and $\sigma^{2}$ was the variance of the composite variable [36]. Model fit was evaluated by the R-squared value of the endogenous variable. Proportion of maximum possible (POMP) scores in the endogenous variable with per unit change in the exogenous variable was calculated to assess effect size: [parameter estimate/(maximum scale value - minimum scale value) +1$)] \star 100$ [37].

\section{Results}

\section{Demographics}

Table 1 presents participants' demographic characteristics $(N=338,212$ intervention and 126 comparison). Mean age was 29.39 years $(S D=4.93)$, and mean postpartum period was 1.76 years $(S D=$ 1.27). Most women were non-smokers (70.4\%) and had some college or less education (75.74\%). Over $40 \%$ were employed full- or part-time $(41.42 \%)$. There were significant between-group differences in postpartum period $(p=0.01)$ and employment status $(p=0.006)$. 
Table 1

\begin{tabular}{|c|c|c|c|}
\hline Age (years) & $29.21(4.92)$ & $29.63(4.95)$ & 0.44 \\
\hline Postpartum period (years) & $1.63(1.23)$ & $1.99(1.30)$ & 0.01 \\
\hline Race & & & 0.08 \\
\hline Non-Hispanic White & $179(84.43 \%)$ & $97(76.98 \%)$ & \\
\hline Non-Hispanic Black & $33(15.57 \%)$ & $29(23.02 \%)$ & \\
\hline Smoking & & & 0.28 \\
\hline Non-smoker & $175(82.55 \%)$ & $98(77.78 \%)$ & \\
\hline Smoker & $37(17.45 \%)$ & $28(22.22 \%)$ & \\
\hline Education & & & 0.47 \\
\hline High school or less & $59(27.83 \%)$ & $43(34.12 \%)$ & \\
\hline Some college or technical school & $100(47.17 \%)$ & $54(42.86 \%)$ & \\
\hline College graduate or higher & $53(25.00 \%)$ & $29(23.02 \%)$ & \\
\hline Employment status & & & 0.006 \\
\hline Full-time & $34(16.04 \%)$ & $32(25.40 \%)$ & \\
\hline Part-time & $46(21.70 \%)$ & $28(22.22 \%)$ & \\
\hline Unemployed & $28(13.21 \%)$ & $28(22.22 \%)$ & \\
\hline Homemaker & $79(37.26 \%)$ & $28(22.22 \%)$ & \\
\hline Self-employed, student and other & $25(11.79 \%)$ & $10(7.94 \%)$ & \\
\hline
\end{tabular}

\section{Total effects of intervention}

The intervention alleviated depressive symptoms $(B=-2.42, p=0.015, \mathrm{POMP}=-4.31 \%$; Table 2$)$. Rsquared value was 0.57 , meaning the whole mediation model explained about $57 \%$ of the variance in depressive symptoms. 
Table 2

Direct and indirect effects of mediation testing while adjusting for baseline measures and covariates
B (SE)
B $(95 \%$
p-value $B$
POMP

Total Effect of Intervention

Intervention $\diamond$ Depressive symptoms_T2

$-2.42$

-4.37 ,

0.015

$-0.11-4.31 \%$

(1.00) $\quad-0.46$

\section{Direct Effects}

Intervention $\diamond$ Autonomous motivation_T2

0.12

(0.08)

$-0.04$

0.145

$0.07 \quad 1.88 \%$

Intervention $\diamond$ Coping self-efficacy_T2

0.30

$(0.06)$

0.29

Intervention $\diamond$ Emotional coping_T2

0.15
$(0.05)$

$0.19,0.41<0.001$

$0.257 .40 \%$

$(0.06)$

Autonomous motivation_T2 $\diamond$ Depressive

symptoms_T2

1.70

$(0.71)$

Coping self-efficacy_T2 $\diamond$ Depressive

symptoms_T2

$\begin{array}{lllll}-4.80 & -7.48, & <0.001 & -0.25 & -8.56 \% \\ (1.37) & -2.12\end{array} \quad$

Emotional coping_T2 $\diamond$ Depressive

symptoms_T2

$-1.77$

(2.82)

$0.05,0.24 \quad 0.003$

$0.18 \quad 3.81 \%$

Indirect Effects (Mediation)

\begin{tabular}{llllll} 
Intervention $\diamond$ Autonomous motivation_T2 $\diamond$ & 0.21 & -0.15, & 0.256 & 0.01 & $0.37 \%$ \\
Depressive symptoms_T2 & $(0.18)$ & 0.57 & & & \\
\hline Intervention $\diamond$ Coping self-efficacy_T2 & -1.42 & -2.33 & 0.002 & -0.06 & $-2.53 \%$ \\
Depressive symptoms_T2 & $(0.46)$ & -0.51 & & & \\
\hline Intervention $\diamond$ Emotional coping_T2 $\diamond$ & -0.26 & -1.10, & 0.555 & -0.01 & $-0.45 \%$ \\
Depressive symptoms_T2 & $(0.43)$ & 0.59 & & &
\end{tabular}

\section{Direct Effect}

$\begin{array}{llllll}\text { Intervention } \diamond \text { Depressive symptoms_T2 } & \begin{array}{l}-0.95 \\ (1.02)\end{array} & \begin{array}{l}-2.95, \\ 1.06\end{array} & 0.355 & -0.04 & -1.68 \%\end{array}$

\section{Other Paths}

\begin{tabular}{|c|c|c|c|c|c|}
\hline Postpartum $\diamond$ Depressive symptoms_T2 & $\begin{array}{l}0.48 \\
(0.35)\end{array}$ & $-0.21,1.17$ & 0.171 & 0.06 & $0.85 \%$ \\
\hline $\begin{array}{l}\text { Depressive symptoms_T1 } \diamond \text { Depressive } \\
\text { symptoms_T2 }\end{array}$ & $\begin{array}{l}0.61 \\
(0.07)\end{array}$ & $0.47,0.76$ & $<0.001$ & 0.57 & $1.09 \%$ \\
\hline $\begin{array}{l}\text { Autonomous motivation_T1 } \diamond \text { Autonomous } \\
\text { motivation_T2 }\end{array}$ & $\begin{array}{l}0.65 \\
(0.07)\end{array}$ & $0.51,0.78$ & $<.001$ & 0.65 & $9.90 \%$ \\
\hline
\end{tabular}




\begin{tabular}{|llllll|}
\hline & B (SE) & $\begin{array}{l}\text { B }(95 \% \\
\text { Cl) }\end{array}$ & p-value & B & POMP \\
\hline $\begin{array}{l}\text { Coping self-efficacy_T1 } \diamond \text { Coping self- } \\
\text { efficacy_T2 }\end{array}$ & $\begin{array}{l}0.79 \\
(0.08)\end{array}$ & $0.64,0.95$ & $<$ & 0.65 & $19.77 \%$ \\
\hline Emotional coping_T1 $\diamond$ Emotional coping_T2 & $\begin{array}{l}0.80 \\
(0.09)\end{array}$ & $0.61,0.98$ & $<$ & 0.001 & \\
\hline
\end{tabular}

\section{Direct and indirect (mediation) effects}

Table 2 presents the results of direct and indirect effects while adjusting for baseline measures and postpartum period. Figure 2 presents the significant direct and indirect paths of the mediation testing.

While the intervention did not significantly increase autonomous motivation, it increased coping selfefficacy $(B=0.30, p<0.001$, POMP $=7.4 \%)$ and emotional coping $(B=0.15, p=0.003, \mathrm{POMP}=3.81 \%)$. When controlling for the intervention, autonomous motivation was positively associated with depressive symptoms $(\mathrm{B}=1.70, p=0.017$, $\mathrm{POMP}=3.02 \%)$. Coping self-efficacy was negatively associated with depressive symptoms $(B=-4.8, p<0.001, \mathrm{POMP}=-8.56 \%)$, but emotional coping was not significantly associated with depressive symptoms.

When assessing the potential role of autonomous motivation and emotional coping as mediators, neither autonomous motivation nor emotional coping significantly mediated the effect of the intervention on depressive symptoms. However, coping self-efficacy negatively mediated the association between the intervention and depressive symptoms $(\mathrm{B}=-1.42, p=0.002$, POMP $=-2.53 \%)$. When controlling for the indirect effects of the intervention on depressive symptoms through autonomous motivation, coping selfefficacy, and emotional coping, the intervention had no significant influence on depressive symptoms. The findings support that coping self-efficacy was the only mediator, but autonomous motivation and emotional coping had no influence on the association between the intervention and depressive symptoms.

\section{Discussion}

To our knowledge, this study is the first to show that coping self-efficacy, but not autonomous motivation or emotional coping, may be responsible for reducing depressive symptoms following a lifestyle behavior intervention for low-income overweight and obese women with young children. The finding that coping self-efficacy significantly mediated the association between the intervention and depressive symptoms is inconsistent with results from another RCT that showed neither problem-focused coping, problem-solving ability, nor social coping mediated the effect of a problem-solving education intervention on depression among low-income mothers with young children [38]. The contradictory findings might have resulted from differences between the studies in participant demographics and measurement. While the current study included predominately Non-Hispanic White overweight and obese women, the previous study included predominately Hispanic women with all body sizes [38]. The current study measured coping selfefficacy, whereas the prior study measured mastery or the degree that women perceived themselves as 
being in control of their lives [38]. Even though the current study showed a small effect size (POMP = $-2.53 \%)$ for coping self-efficacy, it underscored the importance of including coping self-efficacy in intervention studies aimed at alleviating depressive symptoms in low-income overweight and obese women with young children [39].

The current study demonstrated that the lifestyle behavior intervention alleviated depressive symptoms. This finding is consistent with results of a prior systematic review and meta-analysis of five RCTs conducted with overweight or obese women of reproductive age who participated in lifestyle behavior interventions [16]. Four of the five interventions tested in the RCTs were directed toward improving both PA and diet, and one focused only on increasing PA. However, in contrast to the current study, none of the five interventions had a stress management component. Moreover, none of the RCTs specifically focused on only low-income women with young children; therefore, the findings cannot be generalized to this highrisk group. Only one study was found that included predominately low-income women to test the effect of a lifestyle behavior intervention on reducing depressive symptoms. The culturally-tailored intervention, which was designed to increase PA and improve diet among Latina pregnant or postpartum women, consisted of home visits and group meetings that were conducted during pregnancy and between two and six weeks postpartum. Compared to the control, the intervention group had a significantly greater decrease in depressive symptoms from baseline to late pregnancy; however, from baseline to approximately six weeks postpartum, no between-group differences occurred [40]. Although continued research is needed in this area, the findings suggest that interventions addressing only PA and/or diet may not be adequate for achieving this outcome in low-income women with young children.

The positive intervention effect on coping self-efficacy supports results from both a mind-body intervention for human immunodeficiency virus (HIV)-infected individuals [41] and a self-directed cognitive behavioral therapy and mindfulness-based stress reduction intervention for adults [42]. Similar to the current study, these two studies included practical problem-solving strategies (e.g., time management; realistic goal setting) to overcome daily challenges in order to manage stress [26, 43]. Researchers aiming to increase coping self-efficacy may want to consider including practical problemsolving strategies in future studies.

Consistent with a prior study of young women [22], this study found that increasing coping self-efficacy reduced depressive symptoms. The findings support Bandura's [44] Social Cognitive Theory propositions that high coping self-efficacy can mitigate depressive symptoms. Some examples of strategies to increase coping self-efficacy are modeling appropriate behaviors, providing feedback on progress to promote mastery, using verbal persuasion through encouraging statements [44], helping individuals to identify strengths, recognizing existing skills, and encouraging small steps to change [24].

The finding that this study's intervention increased emotional coping was consistent with results from a RCT that included family caregivers of individuals with dementia [45]. Both the current and prior studies focused on teaching participants relaxation exercises (e.g., breathing deeply, counting to 10 , and taking a walk) [24] and emotion-focused coping strategies (e.g., thinking positively and seeking emotional 
support). Therefore, relaxation exercises and emotion-focused coping strategies may warrant consideration to increase emotional coping.

Results from the current study showing that emotional coping had no influence on depressive symptoms were consistent with those from a mindfulness-based intervention to reduce overeating in low- to middleincome overweight pregnant women [46]. The similar findings suggest that emotion-focused coping strategies may be insufficient for reducing depressive symptoms in overweight and obese women who have a low-income.

The lack of an intervention effect on autonomous motivation in this study contrasted with results from a PA intervention for overweight and obese women [47]. The inconsistent findings may have occurred due to measurement issues. To measure autonomous motivation, the current study included the Treatment Self-Regulation Questionnaire [30], whereas the latter study used the behavior-specific Exercise SelfRegulation Questionnaire [47]. Regardless, this study's lack of an intervention effect on autonomous motivation was not completely surprising because of low attendance in the peer support group teleconferences (average 2.6 [SD $=3.4$ ] of 10 teleconferences; $12.4 \%$ completed all 10 ). Despite efforts to schedule teleconferences at times convenient for each group's members, women's busy schedules, sudden time conflicts, or disconnected phones prevented them from attending some sessions [27]. Thus, the inability to receive the expected dose of motivational interviewing may have hampered the intervention effect on autonomous motivation.

The positive association between autonomous motivation and depressive symptoms in this study is counter to Self-Determination Theory and evidence indicating that autonomous motivation contributes to enhancing positive mental health outcomes [48-50]. However, one prior study did show that greater intrinsic motivation was positively related to depressive symptoms among athletes playing team sports, the majority of whom were female [51]. One potential explanation for the positive association is that individuals with more depressive symptoms are likely to experience greater psychosocial demands, which may result in increased autonomous motivation to meet the demands [52]. This information suggests that that the association between autonomous motivation and depression may be more complex than anticipated [51], warranting further investigation.

\section{Strengths and limitations}

The study had strengths and limitations. Strengths included its sample of socioeconomically disadvantaged overweight and obese women with young children, a group notably underrepresented in RCTs [53-54]. Because the current study only included overweight and obese mothers of young children in the Midwestern U.S., the findings may not be generalizable to healthy weight mothers and women in other geographical regions. The depressive symptoms data were obtained via self-report instead of clinician interview (or electronic health record), the latter of which is the gold standard for diagnosing a mental health condition [53]. However, the CES-D is the most commonly used measure for depressive symptoms, and conducing clinician interviews to collect data on depressive symptoms is not practical in 
community settings. Also, this study was a secondary analysis, which might have been underpowered to detect some significant findings.

\section{Conclusion}

Findings support the importance of increasing coping self-efficacy in interventions to alleviate depressive symptoms in low-income overweight and obese women with young children. Teaching women practical strategies that can be applied to daily life may be one important approach for accomplishing this task. Future research continues to be needed to unravel key psychological mechanisms through which interventions decrease depressive symptoms.

\section{Abbreviations}

a: Cronbach's alpha, BMI: body mass index; CES-D: Center for Epidemiologic Studies Depression Scale; CISE: composite indicator structural equation; DVD: Digital Video Disc; human immunodeficiency virus: HIV; POMP: proportion of maximum possible; PA: physical activity; RCT: randomized controlled trial; SD: standard deviation; T: time; US: United States; WIC: Women, Infants, and Children.

\section{Declarations}

\section{Acknowledgments}

We would like to acknowledge Ms. Maria Cotts, Michigan State University undergraduate nursing student, who reviewed and edited the references included in this paper. We also would like to thank the study participants for their participation.

\section{Authors' contributions}

LBR contributed to the study conceptualization, design, methodology, literature search, and writing of the initial draft and revisions to the manuscript. MC contributed to the study conceptualization, design, methodology, data interpretation, writing of the manuscript, and revisions to the manuscript. JL contributed to data interpretation and revisions to the manuscript. RB contributed to the data analysis and interpretation and provided revisions to the manuscript. All authors read and approved the final version.

\section{Funding}

The project was supported by the National Institutes of Health/National Institute of Diabetes and Digestive and Kidney Diseases; Grant Award Number: R18-DK-083934; M. W. Chang (PI). This publication's contents are solely the responsibility of the authors and do not necessarily represent the official views of the National Institutes of Health or the National Institute of Diabetes and Digestive and 
Kidney Disease. ClinicalTrials.gov Identifier NCT01839708; Date of registration: February 28, 2013; Date the first participant was enrolled: September 2012; https://clinicaltrials.gov/ct2/show/NCT01839708

\section{Availability of data and materials}

The datasets generated and/or analyzed during the current study are not publicly available because we are in the stage of data analysis to answer other research questions. However, data are available from the corresponding author based on reasonable request.

\section{Ethics approval and consent to participate}

The study was approved and monitored by the Michigan Department of Community Health and Michigan State University Institutional Review Boards. Participation was voluntary. All participants provided written consent to participate if they met the study criteria and understood the requirements.

\section{Consent for publication}

Our manuscript does not include any individual data or sensitive personal information; therefore, consent for publication is not applicable in this case.

\section{Author details}

${ }^{1}$ College of Nursing, Michigan State University, East Lansing, Michigan, USA

${ }^{2}$ College of Nursing, The Ohio State University, Columbus, Ohio, USA

${ }^{3}$ School of Nursing, University of Wisconsin-Madison, Madison, Wisconsin, USA

\section{References}

1. Shidhaye P, Giri P. Maternal depression: a hidden burden in developing countries. Ann Med Health Sci Res. 2014;4:463-5.

2. Gelaye B, Rondon MB, Araya R, Williams MA. Epidemiology of maternal depression, risk factors, and child outcomes in low-income and middle-income countries. Lancet Psychiatry. 2016;3:973-82.

3. Ertel KA, Rich-Edwards JW, Koenen KC. Maternal depression in the United States: nationally representative rates and risks. J Womens Health (Larchmt). 2011;20:1609-17.

4. Heo M, Pietrobelli A, Fontaine KR, Sirey JA, Faith MS. Depressive mood and obesity in US adults: comparison and moderation by sex, age, and race. Int J Obes (Lond). 2006;30:513-9.

5. de Wit LM, van Straten A, Lamers F, Cuijpers P, Penninx BW. Depressive and anxiety disorders: associated with losing or gaining weight over 2 years? Psychiatry Res. 2015;227:230-7.

6. Knitzer J, Theberge S, Johnson K. Reducing maternal depression and its impact on young children: toward a responsive early childhood policy framework. In: Project Thrive Issue Brief No. 2. New York: 
National Center for Children in Poverty, Columbia University, Mailman School of Public Health; 2008.

7. Fitelson E, Kim S, Baker AS, Leight K. Treatment of postpartum depression: clinical, psychological and pharmacological options. Int J Womens Health. 2010;3:1-14.

8. Faleschini S, Rifas-Shiman SL, Tiemeier H, Oken E, Hivert MF. Associations of prenatal and postnatal maternal depressive symptoms with offspring cognition and behavior in mid-childhood: a prospective cohort study. Int J Environ Res Public Health. 2019;16:e1007.

9. Ulmer-Yaniv A, Djalovski A, Priel A, Zagoory-Sharon O, Feldman R. Maternal depression alters stress and immune biomarkers in mother and child. Depress Anxiety. 2018;35:1145-57.

10. Goodman SH, Garber J. Evidence-based interventions for depressed mothers and their young children. Child Dev. 2017;88:368-77.

11. McDaniel M, Lowenstein C. Depression in low-income mothers of young children: are they getting the treatment they need? Washington, DC: The Urban Institute; 2013. http://www.urban.org. Accessed 18 May 2020.

12. Woolhouse H, Gartland D, Mensah F, Brown SJ. Maternal depression from early pregnancy to 4 years postpartum in a prospective pregnancy cohort study: implications for primary health care. BJOG. 2015;122:312-21.

13. Lee CT, Stroo M, Fuemmeler B, Malhotra R, Østbye T. Trajectories of depressive symptoms over 2 years postpartum among overweight or obese women. Womens Health Issues. 2014;24:559-66.

14. van der Waerden J, Galéra C, Saurel-Cubizolles MJ, Sutter-Dallay AL, Melchior M, the EDEN MotherChild Cohort Study Group. Predictors of persistent maternal depression trajectories in early childhood: results from the EDEN mother-child cohort study in France. Psychol Med. 2015;45:19992012.

15. Horwitz SM, Briggs-Gowan MJ, Storfer-Isser A, Carter AS. Persistence of maternal depressive symptoms throughout the early years of childhood. J Womens Health (Larchmt). 2009;18:637-45.

16. van Dammen L, Wekker V, de Rooij SR, Groen H, Hoek A, Roseboom TJ. A systematic review and meta-analysis of lifestyle interventions in women of reproductive age with overweight or obesity: the effects on symptoms of depression and anxiety. Obes Rev. 2018;19:1679-87.

17. Williams GC, Deci EL, Ryan RM. Building health-care partnerships by supporting autonomy: promoting maintained behavior change and positive health outcomes. In: Suchman AL, HintonWalker P, Botelho R, editors. Partnerships in healthcare: Transforming relational process. Rochester: University of Rochester Press; 1998. pp. 67-87.

18. Vieira PN, Mata J, Silva MN, Coutinho SR, Santos TC, Minderico CS, et al. Predictors of psychological well-being during behavioral obesity treatment in women. J Obes. 2011. doi:10.1155/2011/936153.

19. Bandura A. Social foundations of thought and action: a social cognitive theory. Englewood Cliffs: Prentice-Hall; 1986.

20. Santos HP Jr, Kossakowski JJ, Schwartz TA, Beeber L, Fried El. Longitudinal network structure of depression symptoms and self-efficacy in low-income mothers. PLoS One. 2018;13:e0191675. 
21. Chesney MA, Neilands TB, Chambers DB, Taylor JM. Folkman S. A.

22. validity and reliability. study of the coping self-efficacy scale. Br J Health Psychol.

23. 2006.;11:421-437.

24. Kwasky AN, Groh CJ. Vitamin D, depression and coping self-efficacy in young women: longitudinal study. Arch Psychiatr Nurs. 2014;28:362-7.

25. Obembe OB, Adeyemo S, Ogun OC, ljarogbe GT. The relationship between coping styles and depression among caregivers of children with cerebral palsy in Nigeria, West Africa. Arch Clin Psychiatry. 2019;46:145-50.

26. Chang MW, Nitzke S, Brown R. Mothers In Motion intervention effect on psychosocial health in young, low-income women with overweight or obesity. BMC Public Health. 2019;19:56.

27. U. S. Department of Agriculture: Special supplemental nutrition program for women, infants, and children (WIC). https://www.fns.usda.gov/wic (2019). Accessed 18 May 2020.

28. Chang MW, Nitzke S, Brown R, Resnicow K. A community-based prevention of weight gain intervention (Mothers In Motion) among young low-income overweight and obese mothers: design and rationale. BMC Public Health. 2014;14:280.

29. Chang MW, Brown R, Nitzke S. Results and lessons learned from a prevention of weight gain program for low-income overweight and obese young mothers: Mothers In Motion. BMC Public Health. 2017;17:182.

30. Markland D, Ryan RM, Tobin VJ, Rollnick S. Motivational interviewing and self-determination theory. J Soc Clin Psychol. 2005;24:811-31.

31. Miller WR, Rollnick S. Motivational interviewing: helping people change. 3rd ed. New York: The Guilford Press; 2013.

32. Pelletier LG, Tuson KM, Haddad NK. Client motivation for therapy scale: a measure of intrinsic motivation, extrinsic motivation, and amotivation for therapy. J Pers Assess. 1997;68:414-35.

33. Chang MW, Brown R, Nitzke S. Scale development: factors affecting diet, exercise, and stress management (FADESM). BMC Public Health. 2008;8:76.

34. Radloff LS. The CES-D scale: a self-report depression scale for research in the general population. Appl Psychol Meas. 1997;1:385-401.

35. Becker M, Weinberger T, Chandy A, Schmukler S. Depression during pregnancy and postpartum. Curr Psychiatry Rep. 2016;18:32.

36. McDonald RA, Behson SJ, Seifert CF. Strategies for dealing with measurement error in multiple regression. J Acad Bus Econ. 2005;5:80-97.

37. Hausman JA, Liu H, Luo Y, Palmer C. Errors in the dependent variable of quantile regression models (NBER Working Paper No. 25819). 2019. http://www.nber.org/papers/w25819. Accessed 18 May 2020.

38. Petrescu M. Marketing research using single-item indicators in structural equation models. J Marketing Analytics. 2013;1:99-117. 
39. The problem of units and the Cohen P, Cohen J, Aiken LS, West SG. The problem of units and the.

40. circumstance for POMP. Multivariate Behav Res. 1999;34:315-46.

41. Silverstein M, Cabral H, Hegel M, Diaz-Linhart Y, Beardslee W, Kistin CJ, et al. Problem-solving education to prevent depression among low-income mothers: a path mediation analysis in a randomized clinical trial. JAMA Network Open. 2018;1:e180334.

42. Folkman S, Lazarus RS, Dunkel-Schetter C, DeLongis A, Gruen RJ. Dynamics of a stressful encounter: cognitive appraisal, coping, and encounter outcomes. J Pers Soc Psychol. 1986;50:992-1003.

43. Kieffer EC, Caldwell CH, Welmerink DB, Welch KB, Sinco BR, Guzman JR. Effect of the healthy MOMs lifestyle intervention on reducing depressive symptoms among pregnant Latinas. Am J Community Psychol. 2013;51:76-89.

44. Rodkjaer LO, Laursen T, Seeberg K, Drouin M, Johansen H, Dyrehave C, et al. The effect of a mindbody intervention on mental health and coping self-efficacy in HIV-infected individuals: a feasibility study. J Altern Complement Med. 2017;23:326-30.

45. Silva Almodovar A, Surve S, Axon DR, Cooper D, Nahata MC. Self-directed engagement with a mobile app (Sinasprite) and its effects on confidence in coping skills, depression, and anxiety: retrospective longitudinal study. JMIR Mhealth Uhealth. 2018;6:64.

46. Christmann CA, Hoffmann A, Bleser G. Stress management apps with regard to emotion-focused coping and behavior change techniques: a content analysis. JMIR Mhealth Uhealth. 2017;5:e22.

47. Bandura A. Self-efficacy: the exercise of control. New York: W. H. Freeman \& Company; 1997.

48. Li R, Cooper C, Barber J, Rapaport P, Griffin M, Livingston G. Coping strategies as mediators of the effect of the START (strategies for RelaTives) intervention on psychological morbidity for family carers of people with dementia in a randomised controlled trial. J Affect Disord. 2014;168:298-305.

49. Vieten C, Laraia BA, Kristeller J, Adler N, Coleman-Phox K, Bush NR, et al. The mindful moms training: development of a mindfulness-based intervention to reduce stress and overeating during pregnancy. BMC Pregnancy Childbirth. 2018;18:201.

50. Ryan RM, Connell JP. Perceived locus of causality and internalization: examining reasons for acting in two domains. J Pers Soc Psychol. 1989;57:749-61.

51. Deci EL, Ryan RM. Facilitating optimal motivation and psychological well-being across life's domains. Can Psychol. 2008;49:14-23.

52. Huang Y, Lv W, Wu J. Relationship between intrinsic motivation and undergraduate students' depression and stress: the moderating effect of interpersonal conflict. Psychol Rep. 2016;119:52738.

53. Rouse PC, Ntoumanis N, Duda JL, Jolly K, Williams GC. In the beginning: role of autonomy support on the motivation, mental health and intentions of participants entering an exercise referral scheme. Psychol Health. 2011;26:729-49. 
54. Sheehan RB, Herring MP, Campbell MJ. Associations between motivation and mental health in sport: a test of the hierarchical model of intrinsic and extrinsic motivation. Front Psychol. 2018;9:707.

55. Newman HJ, Howells KL, Fletcher D. The dark side of top level sport: an autobiographic study of depressive experiences in elite sport performers. Front Psychol. 2016;7:868.

56. Association of changes in mental health

Alhalel N, Schueller SM, O'Brien MJ. Association of changes in mental health.

57. with weight loss. during intensive lifestyle intervention: does the timing matter? Obes Sci Pract. 2018;4:153-8.

58. UyBico SJ, Pavel S, Gross CP. Recruiting vulnerable populations into research: a systematic review of recruitment interventions. J Gen Intern Med. 2007;22:852-63.

\section{Figures}




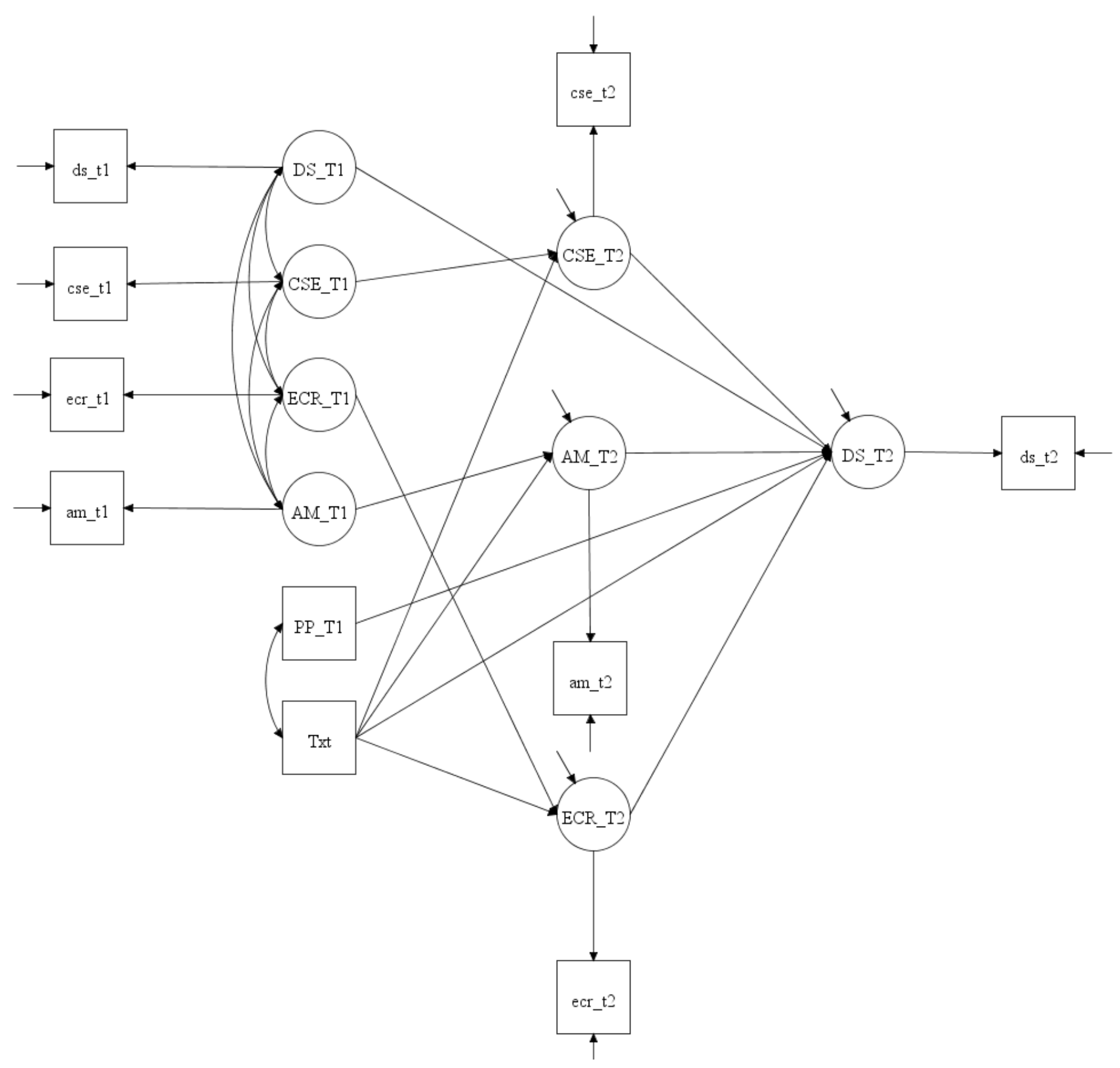

Figure 1

Structure of mediation model testing 


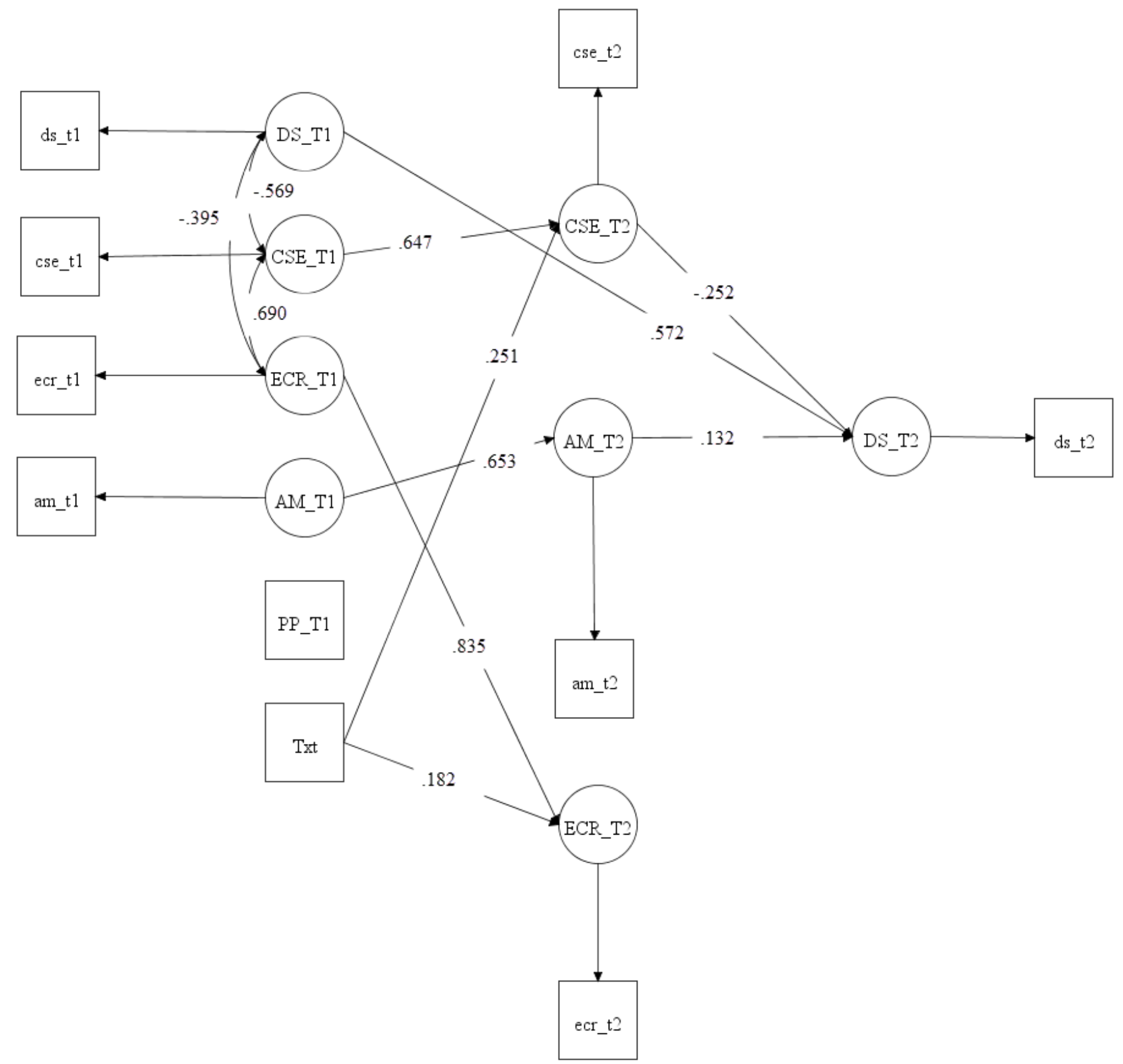

Figure 2

Significant paths of mediation model adjusting for baseline measures and covariate 